\title{
New Antigen Receptor (NARV) Nanobodies: New Promise for Therapeutic Antibodies
}

\section{Dear Editors,}

Single chain antibodies are used as powerful tool to detect interacting antigen proteins because of their small size, better solubility, strong binding affinity and stability. These small antibodies have access to nonimmunogenic antigenic sites, ${ }^{1}$ which otherwise remain buried in the scaffold. In laboratories, single chain antibodies are produced by recombinant antibody technology in which the variable heavy chain $(\mathrm{vH})$ and variable light chains (vL) specific genes are amplified and cloned in a phagemid vector. The nanobodies are expressed on the surface of the phage as fusion proteins and are able to interact with antigen present in its vicinity. The first naturally occurring single chain antibodies were discovered in camel (Camelus dromedarius) and classified as "camelid". 2 These nanobodies naturally lacked the light chains. The heavy chain of the nanobodies contains the antigen binding sites that is referred as $\mathrm{vHH}$ (to distinguish it from $\mathrm{vH}$ ). ${ }^{3}$ Until recently, $\mathrm{vH}$ antibodies were considered to be the smallest intact antigen-binding fragment $(\sim 15$ kilo daltons). ${ }^{3}$ Cloning of $\mathrm{vHH}$ region from an immunized Llama (Lama glama) in a phagemid vector facilitated the construction of the antibody library and biopanning (screening). The development and screening of these antibodies are used to isolate specific antigen binding clones, which belong to various enzymes including lysozyme, amylase, carbonic anhydrase and lactamase. ${ }^{4}$
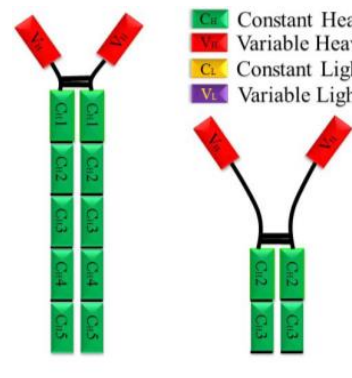

NAR

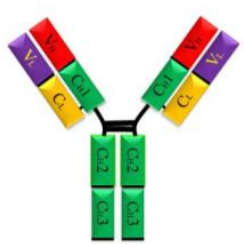

$\mathrm{IgG}$

Fig. 1. Diagram showing the constant $\left(\mathrm{C}_{\mathrm{H}}\right)$ and variable $\left(\mathrm{V}_{\mathrm{H}}\right)$ heavy chains in green and red colors respectively. Constant $\left(\mathrm{C}_{\mathrm{L}}\right)$ and variable $\left(\mathrm{V}_{\mathrm{L}}\right)$ light chains are represented in yellow and violet colors, respectively. The structure of heavy chains of NARV nanobodies is compared with NAR and IgG antibodies.

More recently, another functional immunoglobulin (Ig) superfamily that shares the desirable protein scaffold characteristics has also been isolated from nurse shark (Ginglymostoma cirratum) and wobbegong shark (Orectolobus maculates). These small antibodies are New Antigen Receptor (NAR). ${ }^{5}$ Like camelid antibodies, NAR antibodies also naturally lacks light

chains. The antigen binding sites of these NAR antibodies designated as NARV, which structurally resemble with $\mathrm{vL}$ and $\mathrm{T}$ cell receptor variable regions. ${ }^{6}$ In NARV regions between frame work 2 to CDR2 are deleted tailoring them reduce further to smaller size $(\sim 15$ kilodaltons $)$ and are termed as "Nanobodies". NARVs have become first choice for bioengineering purposes. Since they are even smaller than $\mathrm{vHH}(\sim 15$ kilodaltons $)$ from camels, they are currently considered as the smallest Ig like binding sites found in the animal kingdom. Regular IgG, Llama heavy chain antibody and shark NARV are shown in Fig.1.

Rx Biosciences is actively engaged in custom antibody generation and modulation services. The company provides services to raise polyclonal and monoclonal antibodies, antibody maturation, and humanization and assay development. We generate $\mathrm{scFv}$ and Fab nanobody and IgNAR-V libraries in phage-, bacterial-, yeast-, mRNA- and ribosomal-display systems. In addition, Rx Biosciences provides various cDNA and genomic library constructions, and also sells variety of biological products necessary for the research.

\section{References:}

1. van der Linden RH, et al., Verrips CT: Comparation of physical chemical properties of LlamaVHH antibody fragments and mouse monoclonal antibodies. 1999, Biochim Biophys Acta 1431:37-46.

2. Hamers-Casterman, C, et al., Hamers R: Naturally occuring antibodies devoid of light chains. 1993, Nature 363:446-8.

3. Muyldermans S: Single domain camel antibodies: current status. 2001, Mol Biotechnol 74:277-302.

4. Conrath KE, et al., Muyldermans S: Lactamase inhibitors derived from single-domain antibody fragments elicited in Camelidae. 2001, Antimicrob Agents Chemother 45:2807-12.

5. Dooley H, et al., Porter AJ: Selection and characterization of naturally occuring single-domain (IgNAR) antibody fragments from immunized sharks by phage display. 2003, Mol Immunol 40:25-33.

6. Greenberg AS, et al., Flajnik MF: A new antigen receptor family that undergoes rearrangement and extensive somatic diversification in sharks. 1995, Nature 374:16-173.

Qazi Hamid, CEO

Rx Biosciences

Germantown, 